\title{
Use of the biceps femoris following failed inferior gluteal flap transfer. Case report
}

\author{
W O Thomas MD,${ }^{1}$ G Bjorn Stark MD,${ }^{2} \mathrm{~J}$ O Basadre MD,${ }^{2} \mathrm{~S}$ W Parry MD \\ ${ }^{1}$ Division of Plastic and Reconstructive Surgery, University of South Alabama Medical \\ School, 3401 Medical Park Drive, Building One, Suite 107, Mobile, Alabama, 36693, \\ USA; ${ }^{2}$ Division of Plastic Surgery, Tulane University School of Medicine, New Orleans, \\ Louisiana, USA.
}

Considering the high recurrence rate of pressure ulceration in paraplegic patients, flap procedures to reconstruct a defect should not be at the expense of another possible future flap. The posterior thigh fasciocutaneous flap is useful for the coverage of ischial and trochanteric pressure sores; the biceps femoris musculocutaneous flap is a useful choice for deep ischial defects. However, the cutaneous portions of these two flaps is nearly identical. The previous transfer of the biceps flap excludes the further use of the posterior thigh flap. In contrast, use of the posterior thigh flap still permits the employment of a biceps V-Y advancement flap. Even if the donor site of the previous posterior thigh flap must be skin-grafted, the graft will remain viable on its muscular bed and function as the cutaneous portion of the flap; thus stable coverage is provided, despite previous use of 'first line' flaps. We demonstrate how careful planning of the stages of flap procedures can allow the most economic use of donor areas in this difficult patient group.

Keywords: pressure sore; posterior thigh flap; biceps femoris flap; musculocutaneous flaps; fasciocutaneous flaps.

\section{Introduction}

The most common areas of pressure sores in paraplegic patients are the soft tissues overlying the bony prominences of the pelvic region: the sacrum, the ischial bones, and the greater trochanter. The surgical treatment of decubitus ulcers has been considerably improved by the application of muscle, musculocutaneous, and fasciocutaneous flaps: the tensor fascia lata, the vastus lateralis, and the rectus femoris muscles (all supplied by the lateral femoral circumflex artery); the gluteus maximus muscle (superior and/or inferior gluteal artery); the inferior gluteal fasciocutaneous flap (inferior gluteal artery or first perforating branch of the deep femoral artery); and the biceps femoris advancement musculocutaneous flap (multiple branches from the deep femoral artery). ${ }^{1,2}$

Commonly, the problems of coverage in this patient group are complex. Multiple ulcers may need to be addressed or patients seen after multiple previous surgeries. Be- cause of the known high recurrence rate of pressure sores in the same location or at new sites, any surgery should be 'conservative' in the sense so as not to disturb donor tissue for future reconstructive procedures (by interfering with the axial pedicles of future flaps).

Whereas the muscles receive an exclusive blood supply from their respective pedicles, the overlying skin often has multiple overlapping patterns of perfusion from perforators of neighboring muscle groups and/or fasciocutaneous areas, as well as direct cutaneous vessels. If a fasciocutaneous flap is used and the donor site over an axial muscle skin grafted, this muscle can still be used later as a musculocutaneous flap, with the grafted area representing its cutaneous portion.

\section{Case report}

A 38 year old patient had been paraplegic after a gunshot wound to the cervical spinal 
cord in 1979. To cover a pressure sore of his sacrum he had bilateral V-Y gluteus advancement in 1984. A right tensor fascia lata myocutaneous flap and a posterior thigh (inferior gluteal) fasciocutaneous flap were transferred to the left trochanter in February of 1989 . The large donor site area of the posterior thigh flap measuring $15 \times 32 \mathrm{~cm}$ overlying the biceps femoris muscle was skin grafted. This resulted in stable trochanteric coverage and complete take of the skin graft.

He was readmitted in July of 1989 for treatment of a deep ischial pressure sore with an infected left hip joint and a deep $10 \times 15 \mathrm{~cm}$ ulcer over the right ischium. A staged procedure was performed: the left proximal femur was resected in a Girdlestone procedure and the left vastus lateralis was used as a muscle flap to obliterate the infected cavity. The muscle was covered with a meshed split thickness skin graft. At the same time a debridement of the right ischial sore was performed. The muscle flap healed in completely without signs of generalized infection though about $60 \%$ of the skin graft was lost.

Four weeks later, on September 15, 1989, the right ischial ulcer was debrided again and a partial ischiectomy performed to remove the bony pressure point. This resulted in a deep cavity. Because of the previous surgery for the right trochanteric ulcer, the posterior thigh flap and the tensor fascia lata on the right were not available. Also, because of the prior use of the tensor fascia lata, transfer of the vastus lateralis and rectus femoris muscles, which share the origin of their axial pedicles with the tensor fascia lata in the lateral femoral circumflex artery, was considered unsafe. The complete skin island of the biceps femoris muscle flap had been taken as the inferior gluteal (posterior thigh) fasciocutaneous flap. The biceps vasosome had been replaced by a completely healed and stable skin graft.

It was decided to use this grafted area based on the biceps femoris muscle as a V-Y advancement musculocutaneous flap to cover the defect, as classically described for this muscle with the intact overlying skin supplied by musculocutaneous perforators. ${ }^{3}$
The proximal biceps muscle was used to obliterate the ischial cavity and the overlying previously grafted skin provided external coverage. Primary closure of the donor defect was possible. At the same time, a regrafting of the left vastus lateralis muscle flap was performed. Postoperative healing of the advancement flap was uneventful and 8 weeks after discharge the patient still has stable coverage of his right ischial area, although sitting most of the day (Figs 1-4).

\section{Discussion}

The biceps femoris V-Y musculocutaneous flap provides sufficient proximal muscle bulk to obliterate large ischial ulcers. ${ }^{4,5}$ The overlying skin area supplied by the musculocutaneous perforating vessels is even larger than the muscle. This skin area has a nearly identical distribution to the posterior thigh (inferior gluteal) fasciocutaneous

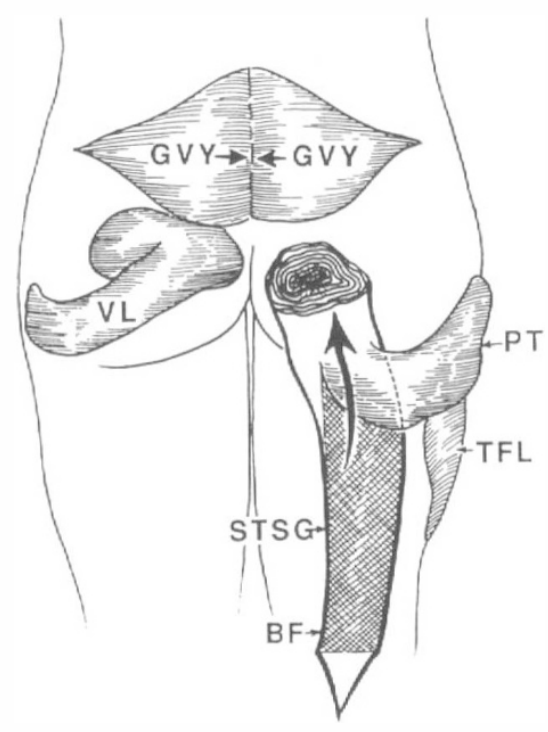

Figure 1 A schematic illustration of previously used flaps and the pregrafted biceps musculocutaneous flap (BF), bilateral V-Y gluteus advancement for a sacral pressure sore (GVY), right tensor fascia lata (TFL), and posterior thigh (PT) flaps for an ischial pressure sore, and a left vastus lateralis (VL) muscle flap to obliterate the cavity after a Girdlestone procedure. 


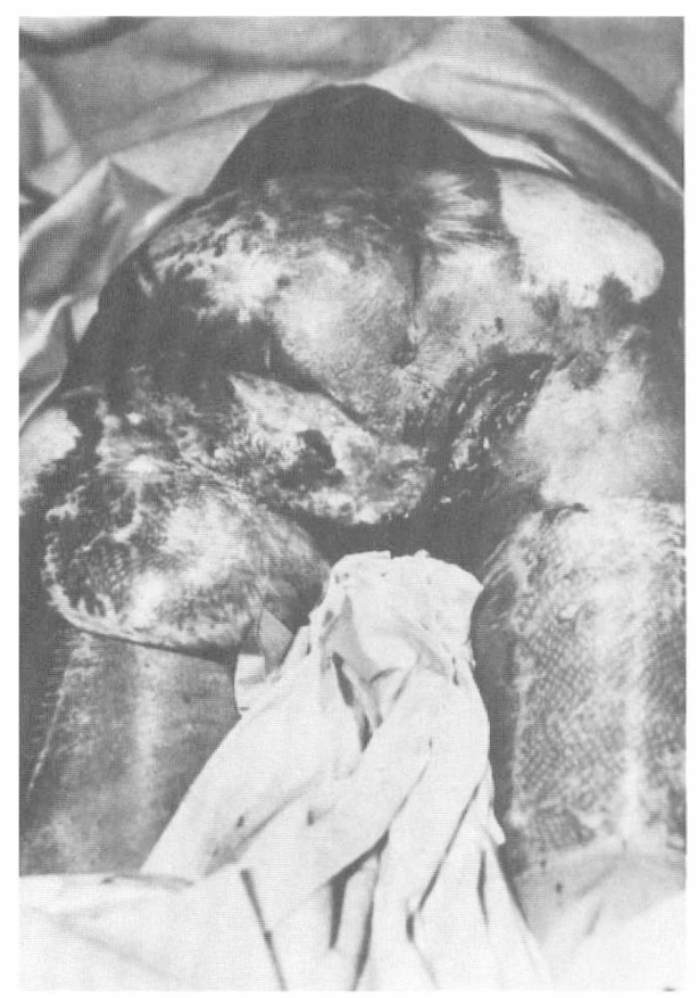

Figure 2 Intraoperative view after debridement of the right ischium. The patient recently had a left vastus lateralis muscle transfer to the ischium, with an incomplete take of the skin graft. On the right posterior thigh the skin graft overlying the area of the biceps femoris after inferior gluteal fasciocutaneous flap transfer to the right trochanter can be seen.

flap. ${ }^{6}$ Thus, the skin of the posterior proximal two thirds of the thigh has a dual blood supply allowing its transfer as a proximally based fasciocutaneous flap or as a musculocutaneous flap.

The biceps femoris V-Y myocutaneous flap is used primarily for ischial sores. In our hands, it is the musculocutaneous flap of choice for the isolated ischial sore so that the gluteus maximus musculocutaneous flap $^{7}$ may be used for subsequent sacral coverage problems. However, the segmental vasculature of the biceps muscle limits its applicability as an island flap. The biceps V-Y advancement excludes the further use of the ipsilateral posterior thigh

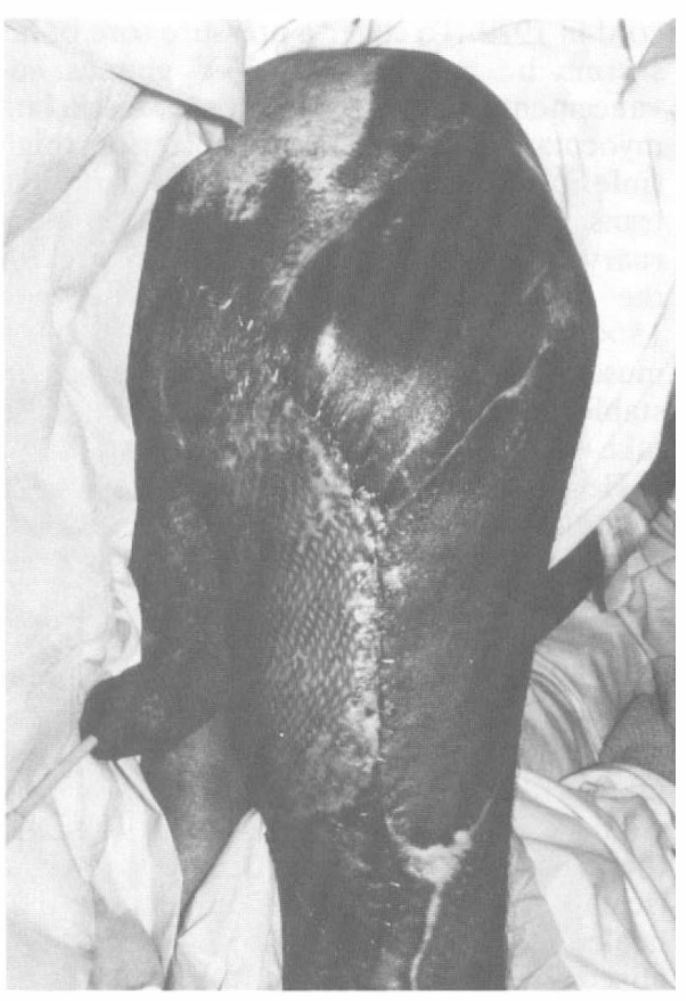

Figure 3 Early postoperative view (POD \#6) after V-Y advancement of the right biceps femoris muscle with the overlying skin graft to cover the right ischium. The left vastus lateralis graft was regrafted at the same time.

skin as a fasciocutaneous flap based on the inferior gluteal artery, for the pedicle of this flap has to be sacrificed with the skin incisions for the biceps flap.

Our case demonstrates that careful planning can ensure that both flaps can be utilized if the sequence of the procedures is reversed: skin grafting of the posterior thigh donor defect overlying the biceps femoris muscle creates a new skin coverage for this muscle which can be used in the usual fashion as a muscle-skin graft flap. This experience emphasizes the staging of flap procedures for pressure sore coverage in paraplegics in an economic manner: by using fasciocutaneous flaps as primary treatment and grafting the underlying muscles these still can be used later as myocutaneous flaps. 


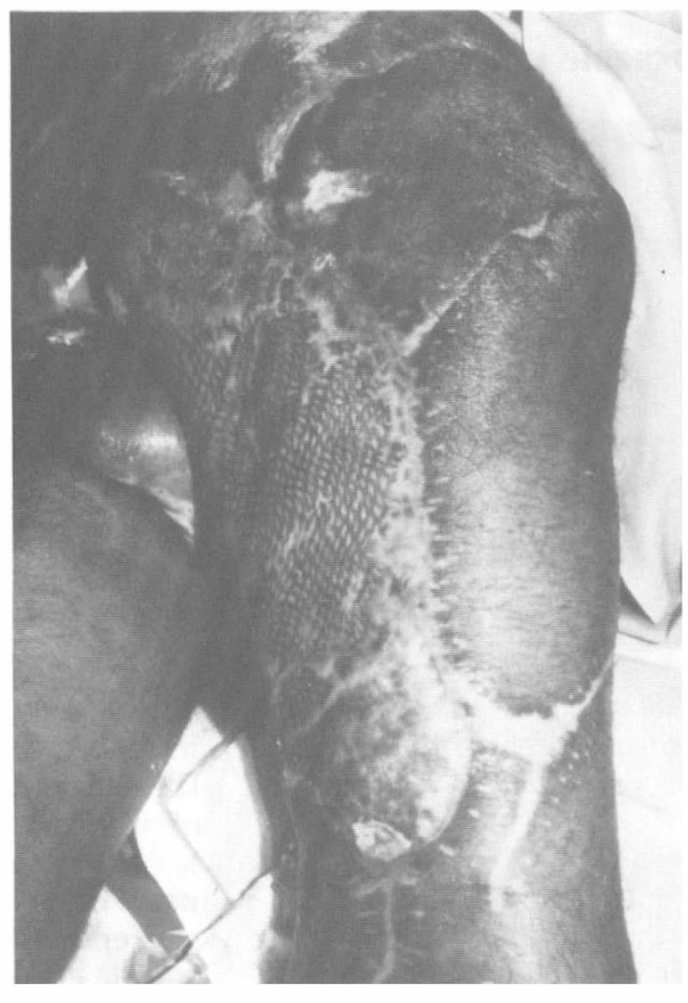

Figure 4 Eight weeks postoperatively the obliteration of the right ischial cavity with the biceps muscle covered previously with a graft results in stable coverage.

\section{References}

1 Hagerty RF, Hagerty HG, Hagerty HF (1980) The hamstring musculocutaneous flap in repair of ischial decubiti. Ann Plast Surg 5: 227-231.

2 Hurteau JE, Bostwick J, Nahai F, Hester R, Jurkewicz MJ (1981) V-Y advancement of hamstring musculocutaneous flap for coverage of ischial pressure sores. Plast Reconstr Surg 68: 539-542.

3 McGraw JB, Arnold PG (1986) McGraw and Arnold's Atlas of Muscle and Musculocutaneous Flaps. Hampton Press, Norfolk, Virginia: 377-383.

4 Tobin GR, Sanders BP, Man D, Weiner LJ (1981) The biceps femoris myocutaneous advancement flap: a useful modification of ischial pressure ulcer reconstruction. Ann Plast Surg 6: 396-401.

5 Scheflan M (1982) Surgical methods for managing ischial pressure wounds. Ann Plast Surg 8: 238-247.

6 Hurwitz DJ, Swartz WM, Mathes SJ (1981) The gluteal thigh flap: a reliable sensate flap for the closure of buttock and perineal wounds. Plast Reconstr Surg 68: 521-532.

7 Ramirez OM (1987) The distal gluteus maximus advancement musculocutaneous flap for coverage of trochanteric pressure sores. Ann Plast Surg 18: 295-302. 